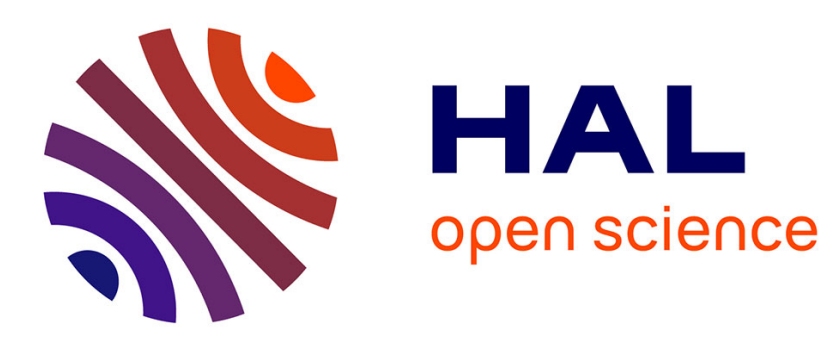

\title{
The reduced scalar potential in regions with permeable materials: Reasons for loss of accuracy and cancellation
}

\author{
Stéphane Balac, Gabriel Caloz
}

\section{To cite this version:}

Stéphane Balac, Gabriel Caloz. The reduced scalar potential in regions with permeable materials: Reasons for loss of accuracy and cancellation. International Journal of Numerical Modelling: Electronic Networks, Devices and Fields, 2007, 20 (4), pp.163-180. 10.1002/jnm.638 . hal-01163782

\author{
HAL Id: hal-01163782 \\ https://hal.science/hal-01163782
}

Submitted on 7 Dec 2015

HAL is a multi-disciplinary open access archive for the deposit and dissemination of scientific research documents, whether they are published or not. The documents may come from teaching and research institutions in France or abroad, or from public or private research centers.
L'archive ouverte pluridisciplinaire HAL, est destinée au dépôt et à la diffusion de documents scientifiques de niveau recherche, publiés ou non, émanant des établissements d'enseignement et de recherche français ou étrangers, des laboratoires publics ou privés. 


\title{
The reduced scalar potential in regions with permeable materials : reasons for loss of accuracy and cancellation
}

\author{
Stéphane Balac \\ Institut Camille Jordan (UMR CNRS 5208) \\ INSA de Lyon, 69621 Villeurbanne, France \\ and \\ Gabriel Caloz \\ Institut de Recherche Mathématique de Rennes (UMR CNRS 6625) \\ Université de Rennes 1, 35042 Rennes, France
}

\begin{abstract}
Practical three-dimensional magnetic field problems usually involve regions containing current sources as well as regions with magnetic materials. For computational purposes the use of the reduced scalar potential (RSP) as unknown has the advantage to transform a problem for a vector field throughout the space into a problem for a scalar function, thus reducing the number of degrees of freedom in the discretization. However in regions with high magnetic permeability the use of the RSP alone usually results in severe loss in accuracy and it is recommended to use both the reduced scalar potential and the total scalar potential. Using an asymptotic expansion, we investigate theoretically the underlying reasons for this lack of accuracy in permeable regions when using the RSP as a unique potential. Moreover this investigation leads to an efficient numerical method to compute the magnetic field in regions with high magnetic permeability.
\end{abstract}

\section{INTRODUCTION}

Practical magnetic field problems usually involve regions containing current sources as well as regions with magnetic materials. It is well known that the use of the reduced scalar potential (RSP) as a single potential for magnetic field computations gives important loss in accuracy in regions with high magnetic permeability, see [1], [2], [3]. The magnetic field is computed from the RSP adding the source field to the gradient of the RSP. The lack of accuracy can be explained in regions with high magnetic permeability from numerical experiments: these two quantities are very close in magnitude but have different signs. In order to investigate the underlying reasons of this cancellation we consider the classical problem of the computation of the magnetic field $\mathbf{H}$ generated by an electromagnetic device composed of a weakly ferromagnetic core $\Omega$ and an inductor $\Omega_{s}$ characterized by a time independent current density $\mathbf{j}$ in three dimensional geometry. We denote by $C \Omega$ and $C \Omega_{s}$ the complement of the adherence of $\Omega$ and $\Omega_{s}$ in $\mathbb{R}^{3}$ and their boundaries by $\Sigma$ and $\Sigma_{s}$. We assume for simplicity that the metallic core $\Omega$ has a constant relative magnetic permeability $\mu$. The relative magnetic permeability is a quantity greater than 1 ; for a material such as soft iron $\mu$ has typically a value around $10^{3}$. 
The magnetic field $\mathbf{H}$ satisfies the basic equations of magnetostatics:

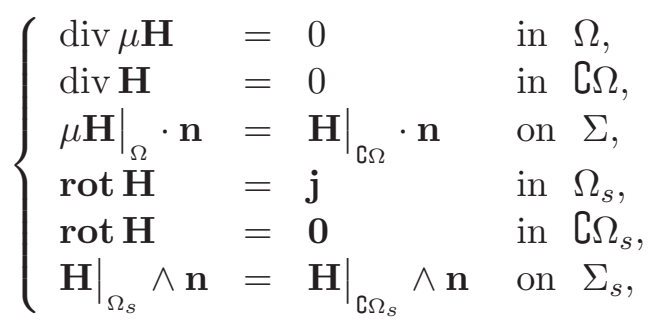

where $\mathbf{n}$ is the outward unit normal to $\Sigma$ or $\Sigma_{s}$, and $\left.\mathbf{H}\right|_{\Omega}\left(\operatorname{resp} .\left.\mathbf{H}\right|_{\mathrm{C} \Omega},\left.\mathbf{H}\right|_{\Omega_{s}},\left.\mathbf{H}\right|_{\mathrm{C} \Omega_{s}}\right)$ denotes the restriction of $\mathbf{H}$ to the domain $\Omega$ (resp. $\complement \Omega, \Omega_{s}, C \Omega_{s}$ ). As $\mathbf{H}$ is not curl free in the domain $\Omega_{s}$ containing the currents, it is not possible to introduce a scalar potential as unknown in the whole space. A classical way to deal with problem (1) using a scalar potential as unknown is to split it in two problems, see [1],[2],[3]. Namely, the magnetic field is expressed as $\mathbf{H}=\mathbf{H}_{\mathbf{s}}+\mathbf{H}_{\mathbf{m}}$ where $\mathbf{H}_{\mathbf{s}}$, the field due to the source currents, satisfies:

$$
\left\{\begin{aligned}
\operatorname{div} \mathbf{H}_{\mathbf{s}} & =0 & & \text { in } \mathbb{R}^{3} \\
\operatorname{rot} \mathbf{H}_{\mathbf{s}} & =\mathbf{j} & & \text { in } \Omega_{s} \\
\operatorname{rot} \mathbf{H}_{\mathbf{s}} & =\mathbf{0} & & \text { in } C \Omega_{s} \\
\left.\mathbf{H}_{\mathbf{s}}\right|_{\Omega_{s}} \wedge \mathbf{n} & =\left.\mathbf{H}_{\mathbf{S}}\right|_{C \Omega_{s}} \wedge \mathbf{n} & & \text { on } \Sigma_{s}
\end{aligned}\right.
$$

and $\mathbf{H}_{\mathbf{m}}$, the field due to the presence of magnetic material, satisfies:

$$
\left\{\begin{aligned}
\operatorname{rot} \mathbf{H}_{\mathbf{m}} & =\mathbf{0} & & \text { in } \mathbb{R}^{3}, \\
\operatorname{div} \mathbf{H}_{\mathbf{m}} & =0 & & \text { in } \Omega \text { and } C \Omega, \\
\left.\mu \mathbf{H}_{\mathbf{m}}\right|_{\Omega} \cdot \mathbf{n}-\left.\mathbf{H}_{\mathbf{m}}\right|_{\mathrm{C} \Omega} \cdot \mathbf{n} & =(1-\mu) \mathbf{H}_{\mathbf{s}} \cdot \mathbf{n} & & \text { on } \Sigma .
\end{aligned}\right.
$$

The field $\mathbf{H}_{\mathbf{s}}$ can be efficiently computed (sometimes analytically), see [4], [5], through the evaluation of the Biot and Savart integral:

$$
\mathbf{H}_{\mathbf{s}}(x)=\frac{1}{4 \pi} \int_{\Omega_{s}}\left(\mathbf{j}(y) \wedge \frac{x-y}{|x-y|^{3}}\right) \mathrm{d} y \quad \forall x \in \complement \Omega_{s} .
$$

Then it is possible to introduce the reduced scalar magnetic potential (RSP) $\phi$ as unknown to solve problem (3) since the field $\mathbf{H}_{\mathbf{m}}$ is curl free in the whole space. Introducing the RSP transforms problem (3) for the three components vector field $\mathbf{H}_{\mathbf{m}}=-\nabla \phi$ into the following problem for the scalar function $\phi$,

$$
\left\{\begin{array}{c}
\Delta \phi=0 \quad \text { in } \Omega \text { and } C \Omega, \\
\phi \text { continuous on } \Sigma, \\
\left.\mu \frac{\partial \phi}{\partial n}\right|_{\Omega}-\left.\frac{\partial \phi}{\partial n}\right|_{C \Omega}=(\mu-1) g \quad \text { on } \Sigma,
\end{array}\right.
$$

where $g=\mathbf{H}_{\mathbf{S}} \cdot \mathbf{n}$ is considered as a given function since $\mathbf{H}_{\mathbf{S}}$ is computed using (4). Problem (5) is a classical Laplace problem set in the whole space with an interface condition. It can be solved using various numerical methods (finite element method, boundary element method, ... ), see [2], [6], [7] for instance. We refer to [8] for an overview of derivative extraction methods in electromagnetics used to compute $\mathbf{H}_{\mathbf{m}}$ from the RSP $\phi$. The magnetic field $\mathbf{H}$ is then obtained by adding $\mathbf{H}_{\mathbf{m}}$ to the source field $\mathbf{H}_{\mathbf{s}}$ calculated using formula (4). This approach is efficient and accurate in non-magnetic regions or in regions with low magnetic permeability (e.g. regions with dia or paramagnetic materials). However adding 


\begin{tabular}{|c|c|c|c|c|c|}
\hline$\mu$ & $\mathbf{H}_{\mathbf{s}} \|_{2}$ & $\mathbf{H}_{\mathbf{m}} \|_{2}$ & $\mid \mathbf{H}_{\mathbf{s}}+\mathbf{H}_{\mathbf{m}} \|_{2}$ & $\mid \mathbf{H} \|_{2}$ exact & error (\%) \\
\hline 2 & $0.79510^{6}$ & $0.192910^{6}$ & $0.602110^{6}$ & $0.596210^{6}$ & 0.97 \\
\hline 10 & $0.79510^{6}$ & $0.593710^{6}$ & $0.201310^{6}$ & $0.183510^{6}$ & 9.2 \\
\hline $10^{2}$ & $0.79510^{6}$ & $0.749310^{6}$ & $0.456810^{5}$ & $0.231610^{5}$ & 65 \\
\hline $10^{3}$ & $0.79510^{6}$ & $0.769510^{6}$ & $0.255110^{5}$ & $0.237810^{4}$ & 166 \\
\hline $10^{4}$ & $0.79510^{6}$ & $0.771610^{6}$ & $0.234310^{5}$ & $0.238410^{3}$ & 190 \\
\hline
\end{tabular}

Table 1: Comparison of the norms of the magnetic fields as a function of $\mu$.

the two vector fields $\mathbf{H}_{\mathbf{s}}$ and $\mathbf{H}_{\mathbf{m}}$ to compute the magnetic field $\mathbf{H}$ within a region with high magnetic permeability (e.g. region with soft ferromagnetic materials) leads to very inaccurate results. Indeed, if the total field is small (for instance due to a shielding effect of the permeable region), the two components of the fields would tend to be of the same magnitude, but of different sign leading to an oscillating error prone solution for the magnetic field $\mathbf{H}$.

To illustrate this issue table 1 shows the norms of the fields $\mathbf{H}_{\mathbf{s}}, \mathbf{H}_{\mathbf{m}}$ computed using a finite element method, and $\mathbf{H}$ computed by the relation $\mathbf{H}=\mathbf{H}_{\mathbf{m}}+\mathbf{H}_{\mathbf{s}}$ as well as the exact values of $\mathbf{H}$ for increasing values of $\mu$ in a domain chosen to be a ball (see section 4 for the computational details). One can see that the values for $\mathbf{H}_{\mathbf{m}}$ tend to be of the same magnitude as the values for $\mathbf{H}_{\mathbf{s}}$ when $\mu$ increases leading to very inaccurate results for $\mathbf{H}$.

The consequence of this inaccuracy is that in regions with high magnetic permeability the magnetic field has to be computed directly and not from the RSP through the relation $\mathbf{H}=-\nabla \phi+\mathbf{H}_{\mathbf{s}}$. This is usually done, see [1], [2], [3], by introducing the total magnetic potential $\psi$ : since $\mathbf{H}$ is curl-free in $C \Omega_{s}$ there exists a scalar potential $\psi$ such that $\mathbf{H}=$ $-\nabla \psi$. In $\complement \Omega_{s}$ the total magnetic potential $\psi$ satisfies:

$$
\left\{\begin{array}{c}
\Delta \psi=0 \quad \text { in } \Omega \text { and } C \Omega_{s} \backslash \Omega, \\
\left.\mu \frac{\partial \psi}{\partial n}\right|_{\Omega}-\left.\frac{\partial \psi}{\partial n}\right|_{\mathrm{C} \Omega}=0 \quad \text { on } \Sigma .
\end{array}\right.
$$

The total scalar magnetic potential is not defined in the conductors domain $\Omega_{s}$ where $\operatorname{rot} \mathbf{H}_{\mathbf{s}} \neq 0$. The recommended way to proceed is then to compute the magnetic field $\mathbf{H}$ from the RSP $\phi$ in the current sources region and to use the total magnetic potential $\psi$ elsewhere. The space is thus divided by a closed surface $\Gamma$ into two regions $\Omega_{1}$ and $\Omega_{2}$. The first one $\Omega_{1}$ includes the ferromagnetic domain $\Omega$ and possibly part of the free space. In this region the magnetic field $\mathbf{H}$ is curl free and we introduce the total scalar potential $\psi$ such that $\mathbf{H}=-\nabla \psi$. The second region $\Omega_{2}$ is composed of free space and conductors domain $\Omega_{s}$. As the magnetic field $\mathbf{H}$ is not curl free in $\Omega_{s}$ we introduce in $\Omega_{2}$ the reduced scalar potential $\phi$ such that $\mathbf{H}=-\nabla \phi+\mathbf{H}_{\mathbf{s}}$ with $\mathbf{H}_{\mathbf{s}}$ given by (4). At the boundary $\Gamma$ between $\Omega_{1}$ and $\Omega_{2}$ we have to require the continuity of the normal component of the magnetic induction $\mathbf{B}$ and the continuity of the tangential component of the magnetic field $\mathbf{H}$. This leads to the following boundary conditions on $\Gamma$ for $\psi$ and $\phi$

$$
\begin{aligned}
\mu_{1} \frac{\partial \psi}{\partial n} & =\mu_{2} \frac{\partial \phi}{\partial n}-\mu_{2}\left(\mathbf{H}_{\mathbf{s}} \cdot \mathbf{n}\right) \\
\frac{\partial \psi}{\partial t} & =\frac{\partial \phi}{\partial t}-\mathbf{H}_{\mathbf{s}} \cdot \mathbf{t}
\end{aligned}
$$

where $\mathbf{n}$ denotes a unit normal vector to $\Gamma$ and $\mathbf{t}$ denotes an arbitrarily chosen unit vector tangent to $\Gamma$. Relation (8) can be integrated along a path $\gamma$ on $\Gamma$ from a given fixed point 
$x_{0}$ on $\Gamma$ to any general point $x$ on $\Gamma$. We obtain

$$
\phi(x)-\psi(x)=\int_{\gamma} \mathbf{H}_{\mathbf{s}} \cdot \mathbf{d} \mathbf{t}
$$

if at point $x_{0}$ the two potential $\phi$ and $\psi$ (defined up to a constant) are taken to be equal. The line integral in (9) is path-independent because of Stokes' theorem applied to the curl free field $\mathbf{H}_{\mathbf{s}}$.

In this paper we investigate the way the cancellation occurs when the magnetic field $\mathbf{H}$ is computed by adding the two fields $\mathbf{H}_{\mathbf{m}}$ and $\mathbf{H}_{\mathbf{s}}$ in regions with high magnetic permeability $\mu$ in the single potential approach. The study is based on an asymptotic expansion for the RSP. We show that the leading term in the asymptotic development of $\mathbf{H}_{\mathbf{m}}$ in power of $1 / \mu$ is $-\mathbf{H}_{\mathbf{s}}$. For large values of $\mu$ the other terms in the development are below the accuracy level of the computation leading to very inaccurate results when adding the two fields $\mathbf{H}_{\mathbf{m}}$ and $\mathbf{H}_{\mathbf{s}}$ to calculate $\mathbf{H}$. As a by-product of the study we give a method to compute the total field $\mathbf{H}$ within a region with high magnetic permeability directly from the RSP without introducing the total magnetic potential.

The content of the paper is the following: in section 2 we derive some properties of the RSP. We set up an integral formula to compute $\mathbf{H}_{\mathbf{m}}$ from the RSP and do a careful analysis to know the dependence of the RSP on $\mu$. In section 3 we use the results of section 2 to explain why, in regions with high magnetic permeability, adding the source field to the reaction field to compute the total magnetic field leads to very inaccurate results. This investigation also leads to an efficient numerical method to compute the magnetic field in regions involving domains with high magnetic permeability. We compair this numerical method for the RSP to the two potential method and conclude in section 4 with numerical illustrations.

\section{GENERAL PROPERTIES OF THE REDUCED SCALAR POTENTIAL}

\section{$2.1 \quad$ Variational formulation}

In the following, the ferromagnetic core domain $\Omega$ is assumed to be an open, bounded and connected set in $\mathbb{R}^{3}$ with boundary $\Sigma$ Lipschitz continuous. $\mathbb{L}^{2}(\Omega)$ denotes the set of square integrable functions over $\Omega$ and $\mathbb{H}^{1}(\Omega)$ denotes the set of functions in $\mathbb{L}^{2}(\Omega)$ with derivatives in $\mathbb{L}^{2}(\Omega)$. The set $\mathbb{H}^{1}(\Omega)$ is equipped with the norm $\|\cdot\|_{1, \Omega}$ defined for $\zeta \in \mathbb{H}^{1}(\Omega)$ by $\|\zeta\|_{1, \Omega}^{2}=\int_{\Omega} \zeta^{2} \mathrm{~d} x+\int_{\Omega} \nabla \zeta \cdot \nabla \zeta \mathrm{d} x$. We also introduce the Sobolev space

$$
\mathbb{H}^{1 / 2}(\Sigma)=\left\{\zeta: \Sigma \rightarrow \mathbb{R} ;\left.\exists \xi \in \mathbb{H}^{1}(\Omega) \xi\right|_{\Sigma}=\zeta\right\}
$$

endowed with the norm $\|\zeta\|_{\frac{1}{2}, \Sigma}=\inf _{\substack{\left.\xi \in \mathbb{H}^{1}(\Omega) \\ \xi\right|_{\Sigma}=\zeta}}\|\xi\|_{1, \Omega}$. We denote by $\mathbb{H}^{-1 / 2}(\Sigma)$ the dual space of $\mathbb{H}^{1 / 2}(\Sigma)$. To handle functions defined over the unbounded domain $\complement \Omega$ we will use the standard weighted Sobolev space $\mathbb{W}^{1}(\complement \Omega)$ defined by

$$
\mathbb{W}^{1}(\complement \Omega)=\left\{\zeta ; \frac{\zeta}{\sqrt{1+|x|^{2}}} \in \mathbb{L}^{2}(\complement \Omega), \quad \nabla \zeta \in \mathbb{L}^{2}(\complement \Omega)^{3}\right\}
$$

This set is equipped with the norm $|\cdot|_{1, \complement \Omega}$ defined for $\zeta \in \mathbb{W}^{1}(\complement \Omega)$ by $|\zeta|_{1, \complement \Omega}^{2}=\int_{\complement \Omega} \nabla \zeta$. $\nabla \zeta \mathrm{d} x$. We also introduce the space $\mathbb{W}^{1}\left(\mathbb{R}^{3}\right)$ defined in a similarly way. 
We obtain the weak form of problem (5) by the following standard formal procedure: we multiply the differential equation in $\Omega$ and $C \Omega$ by an arbitrary $\varphi \in \mathbb{W}^{1}\left(\mathbb{R}^{3}\right)$, integrate over $\Omega$ and $C \Omega$ and apply Green's theorem to the two integral equalities. Summing up the two equalities (the one in $\Omega$ multiplied by $\mu$ ) and using Neumann interface condition on $\Sigma$ get the following variational formulation for problem (5): find $\phi \in \mathbb{W}^{1}\left(\mathbb{R}^{3}\right)$ such that

$$
\mu \int_{\Omega} \nabla \phi \cdot \nabla \varphi \mathrm{d} x+\int_{\mathcal{\Omega} \Omega} \nabla \phi \cdot \nabla \varphi \mathrm{d} x=(\mu-1) \int_{\Sigma} g \varphi \mathrm{d} \sigma \quad \forall \varphi \in \mathbb{W}^{1}\left(\mathbb{R}^{3}\right) .
$$

For $g \in \mathbb{H}^{-1 / 2}(\Sigma)$ from the Lax-Milgram theorem one can prove that problem (10) has a unique solution in the Sobolev space $\mathbb{W}^{1}\left(\mathbb{R}^{3}\right)$ and this solution is continuous in $\mathbb{R}^{3}$, see [9].

\subsection{Integral representation formula for the RSP}

Let $G$ denote the Green kernel associated with the three-dimensional Laplacian:

$$
G(x, y)=\frac{1}{4 \pi|x-y|} \quad \text { for } \quad x, y \in \mathbb{R}^{3}, x \neq y,
$$

and $G_{n}(x, y)=\nabla_{x} G(x, y) . \mathbf{n}, x \in \Sigma, y \in \mathbb{R}^{3}$, denotes its normal derivative on $\Sigma$. Since the magnetic potential $\phi$ is harmonic in $\Omega$ we have the Green representation formula for $y \in \Omega$, see [7],[9],

$$
\phi(y)=-\left.\int_{\Sigma} \phi\right|_{\Omega}(x) G_{n}(x, y) \mathrm{d} \sigma_{x}+\left.\int_{\Sigma} \frac{\partial \phi}{\partial n}\right|_{\Omega}(x) G(x, y) \mathrm{d} \sigma_{x} .
$$

Let's take $x \in \complement \Omega$ and $y \in \Omega$. We deduce from Green's second identity the relation:

$$
\begin{aligned}
& 0=\int_{C \Omega}\left(\Delta_{x} \phi(x) G(x, y)-\phi(x) \Delta_{x} G(x, y)\right) \mathrm{d} x \\
& =-\int_{\Sigma}\left(\left.\frac{\partial \phi}{\partial n}\right|_{C \Omega}(x) G(x, y)-\left.\phi\right|_{C \Omega}(x) G_{n}(x, y)\right) \mathrm{d} \sigma_{x} .
\end{aligned}
$$

Then we multiply (11) by $\mu$ and add it to (12) to get for $y \in \Omega$ :

$$
\begin{array}{r}
\mu \phi(y)=\int_{\Sigma}\left(\left.\phi\right|_{\mathrm{C} \Omega}(x)-\left.\mu \phi\right|_{\Omega}(x)\right) G_{n}(x, y) \mathrm{d} \sigma_{x} \\
-\int_{\Sigma}\left(\left.\frac{\partial \phi}{\partial n}\right|_{\complement \Omega}(x)-\left.\mu \frac{\partial \phi}{\partial n}\right|_{\Omega}(x)\right) G(x, y) \mathrm{d} \sigma_{x} .
\end{array}
$$

Using the boundary condition on $\Sigma$ in (5), we obtain the following representation formula for $y \in \Omega$ :

$$
\phi(y)=\frac{\mu-1}{\mu} \int_{\Sigma} g(x) G(x, y) \mathrm{d} \sigma_{x}-\frac{\mu-1}{\mu} \int_{\Sigma} \phi(x) G_{n}(x, y) \mathrm{d} \sigma_{x} .
$$

As a consequence, we can express for $y \in \Omega$ the field $\mathbf{H}_{\mathbf{m}}(y)=-\nabla \phi(y)$ as:

$$
\mathbf{H}_{\mathbf{m}}(y)=-\frac{\mu-1}{\mu} \int_{\Sigma} g(x) \nabla_{y} G(x, y) \mathrm{d} \sigma_{x}+\frac{\mu-1}{\mu} \int_{\Sigma} \phi(x) \nabla_{y} G_{n}(x, y) \mathrm{d} \sigma_{x} .
$$

It can also be shown that for $y \in C \Omega$ the field $\mathbf{H}_{\mathbf{m}}$ is given by

$$
\mathbf{H}_{\mathbf{m}}(y)=-(\mu-1) \int_{\Sigma} g(x) \nabla_{y} G(x, y) \mathrm{d} \sigma_{x}+(\mu-1) \int_{\Sigma} \phi(x) \nabla_{y} G_{n}(x, y) \mathrm{d} \sigma_{x} .
$$




\subsection{Dependence of the RSP on $\mu$ in permeable regions}

To understand why the RSP is not well adapted to compute the total magnetic field in a region with high magnetic permeability, let us investigate how the solution $\phi$ to (5) depends on $\mu$ considered as a parameter. We write down an asymptotic expansion of the RSP $\phi$ in power of $1 / \mu$. For convenience $\phi^{i}$ denotes the restriction of the RSP to the interior domain $\Omega$ and $\phi^{e}$ the restriction of the RSP to the exterior domain $C \Omega$. Problem (5) then reads : find $\phi^{i} \in \mathbb{H}^{1}(\Omega)$ and $\phi^{e} \in \mathbb{W}^{1}(\complement \Omega)$ such that

$$
\begin{cases}\Delta \phi^{i}=0 & \text { in } \Omega, \\ \Delta \phi^{e}=0 & \text { in } C \Omega, \\ \phi^{e}=\phi^{i} & \text { on } \Sigma, \\ \frac{1}{\mu} \frac{\partial \phi^{e}}{\partial n}-\frac{\partial \phi^{i}}{\partial n}=\left(\frac{1}{\mu}-1\right) g & \text { on } \Sigma .\end{cases}
$$

Let us look for $\phi^{i}$ and $\phi^{e}$ in the form of :

$$
\phi^{i}=\sum_{k=0}^{+\infty} \frac{1}{\mu^{k}} \phi_{k}^{i} \quad \text { and } \quad \phi^{e}=\sum_{k=0}^{+\infty} \frac{1}{\mu^{k}} \phi_{k}^{e} .
$$

One can easily check that $\phi_{k}^{i} \in \mathbb{H}^{1}(\Omega)$ and $\phi_{k}^{e} \in \mathbb{W}^{1}(\complement \Omega), k \in\{0,1\}$, should be solutions of the following interior Neumann and exterior Dirichlet coupled problems:

$$
\begin{aligned}
& \left(\mathcal{P}_{0}^{i}\right) \quad \begin{cases}\Delta \phi_{0}^{i}=0 & \text { in } \quad \Omega, \\
\frac{\partial \phi_{0}^{i}}{\partial n}=g & \text { on } \Sigma,\end{cases}
\end{aligned}
$$

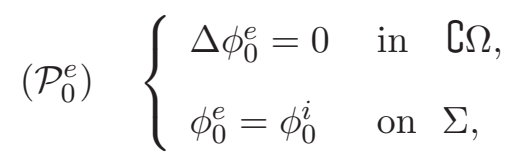

$$
\begin{aligned}
& \left(\mathcal{P}_{1}^{i}\right) \quad \begin{cases}\Delta \phi_{1}^{i}=0 & \text { in } \Omega, \\
\frac{\partial \phi_{1}^{i}}{\partial n}=\frac{\partial \phi_{0}^{e}}{\partial n}-g & \text { on } \Sigma,\end{cases} \\
& \left(\mathcal{P}_{1}^{e}\right) \quad \begin{cases}\Delta \phi_{1}^{e}=0 & \text { in } \quad C \Omega, \\
\phi_{1}^{e}=\phi_{1}^{i} & \text { on } \Sigma .\end{cases}
\end{aligned}
$$

For $k \geq 2, \phi_{k}^{i} \in \mathbb{H}^{1}(\Omega)$ and $\phi_{k}^{e} \in \mathbb{W}^{1}(\complement \Omega)$ should satisfy :

$$
\begin{aligned}
& \left(\mathcal{P}_{k}^{i}\right) \quad \begin{cases}\Delta \phi_{k}^{i}=0 & \text { in } \Omega, \\
\frac{\partial \phi_{k}^{i}}{\partial n}=\frac{\partial \phi_{k-1}^{e}}{\partial n} & \text { on } \Sigma,\end{cases} \\
& \left(\mathcal{P}_{k}^{e}\right) \quad \begin{cases}\Delta \phi_{k}^{e}=0 & \text { in } \quad C \Omega, \\
\phi_{k}^{e}=\phi_{k}^{i} & \text { on } \Sigma .\end{cases}
\end{aligned}
$$

Proposition 1 There exists sequences of functions $\left(\phi_{k}^{i}\right)_{k \in \mathbb{N}},\left(\phi_{k}^{e}\right)_{k \in \mathbb{N}}$ solution to the above coupled problems $\left(\mathcal{P}_{k}^{i}\right)_{k \in \mathbb{N}},\left(\mathcal{P}_{k}^{e}\right)_{k \in \mathbb{N}}$; the solutions are unique. 
Proof. First to have existence for $\left(\mathcal{P}_{0}^{i}\right)$ we need the compatibility condition $\int_{\Sigma} g \mathrm{~d} \sigma=0$ which is satisfied since

$$
\int_{\Sigma} g \mathrm{~d} \sigma=\int_{\Sigma} \mathbf{H}_{\mathbf{s}} \cdot \mathbf{n} \mathrm{d} \sigma=\int_{\Omega} \operatorname{div} \mathbf{H}_{\mathbf{s}} \mathrm{d} x=0 .
$$

Then, there exists a unique $\widetilde{\phi}_{0}^{i} \in\left\{\psi \in \mathbb{H}^{1}(\Omega), \int_{\Omega} \psi \mathrm{d} x=0\right\}$ solution to $\left(\mathcal{P}_{0}^{i}\right)$ and $\phi_{0}^{i}=$ $\widetilde{\phi}_{0}^{i}+C_{0}$ is determined up to a constant. Once $C_{0}$ is known, then $\phi_{0}^{e}$ is uniquely determined by $\left(\mathcal{P}_{0}^{e}\right)$. To have existence for $\left(\mathcal{P}_{1}^{i}\right)$, the compatibility condition then reads $\int_{\Sigma} \frac{\partial \phi_{0}^{e}}{\partial n} \mathrm{~d} \sigma=0$. This condition is satisfied with a convenient choice for $C_{0}$. Let us consider $v \in \mathbb{W}^{1}(C \Omega)$ the unique solution of

$$
\left(\mathcal{P}_{v}\right) \quad \begin{cases}\Delta v=0 & \text { in } \quad C \Omega \\ v=1 & \text { on } \Sigma\end{cases}
$$

Clearly $\int_{\Sigma} \frac{\partial v}{\partial n} \mathrm{~d} \sigma=\int_{\complement \Omega} \nabla v \cdot \nabla v \mathrm{~d} x>0$ and we set:

$$
C_{0}=-\left(\int_{\Sigma} \frac{\partial \widetilde{\phi}_{0}^{e}}{\partial n} \mathrm{~d} \sigma\right) \times\left(\int_{\Sigma} \frac{\partial v}{\partial n} \mathrm{~d} \sigma\right)^{-1}
$$

with $\widetilde{\phi}_{0}^{e}$ given by

$$
\left(\widetilde{\mathcal{P}}_{0}^{e}\right) \quad \begin{cases}\Delta \widetilde{\phi}_{0}^{e}=0 & \text { in } \quad C \Omega, \\ \widetilde{\phi}_{0}^{e}=\widetilde{\phi}_{0}^{i} & \text { on } \Sigma\end{cases}
$$

Then with $\phi_{0}^{e}=\widetilde{\phi}_{0}^{e}+C_{0} v$ the compatibility condition $\int_{\Sigma} \frac{\partial \phi_{0}^{e}}{\partial n} \mathrm{~d} \sigma=0$ is satisfied. Similarly we continue to construct in a unique way the functions $\phi_{k}^{i}$ and $\phi_{k}^{e}$ for $k \geq 1$.

Proposition 2 The series $\sum_{k=0}^{+\infty} \frac{1}{\mu^{k}} \phi_{k}^{i}$ and $\sum_{k=0}^{+\infty} \frac{1}{\mu^{k}} \phi_{k}^{e}$ converge to (the restriction of) $\phi$ in $\mathbb{H}^{1}(\Omega)$ and $\mathbb{W}^{1}(\complement \Omega)$ respectively.

Moreover the following estimates hold, for any integer $N$

$$
\begin{aligned}
&\left|\phi-\sum_{k=0}^{N} \frac{1}{\mu^{k}} \phi_{k}^{e}\right|_{1, \complement \Omega} \leq \frac{C_{e}}{\mu^{N}}\|g\|_{-\frac{1}{2}, \Sigma}, \\
&\left\|\phi-\sum_{k=0}^{N} \frac{1}{\mu^{k}} \phi_{k}^{i}\right\|_{1, \Omega} \leq \frac{C_{i}}{\mu^{N+1}}\|g\|_{-\frac{1}{2}, \Sigma}
\end{aligned}
$$

where $C_{e}, C_{i}$ are positive constants independent of $\mu$, and $\|g\|_{-\frac{1}{2}, \Sigma}$ the norm of $g$ in the dual space of the Sobolev space $H^{1 / 2}(\Sigma)$.

Proof. We set $S_{N}^{i}=\sum_{k=0}^{N} \frac{1}{\mu^{k}} \phi_{k}^{i}$ and $S_{N}^{e}=\sum_{k=0}^{N} \frac{1}{\mu^{k}} \phi_{k}^{e}$. Green's first identity yields

$$
\begin{aligned}
\left|\phi-S_{N}^{e}\right|_{1, \mathrm{C} \Omega}^{2} & =\int_{\complement \Omega} \nabla\left(\phi^{e}-S_{N}^{e}\right) \cdot \nabla\left(\phi^{e}-S_{N}^{e}\right) \mathrm{d} x \\
& =-\int_{\Sigma}\left(\phi^{e}-S_{N}^{e}\right) \frac{\partial}{\partial n}\left(\phi^{e}-S_{N}^{e}\right) \mathrm{d} \sigma
\end{aligned}
$$


and

$$
\begin{aligned}
\left|\phi-S_{N}^{i}\right|_{1, \Omega}^{2} & =\int_{\Omega} \nabla\left(\phi^{i}-S_{N}^{i}\right) \cdot \nabla\left(\phi^{i}-S_{N}^{i}\right) \mathrm{d} x \\
& =\int_{\Sigma}\left(\phi^{i}-S_{N}^{i}\right) \frac{\partial}{\partial n}\left(\phi^{i}-S_{N}^{i}\right) \mathrm{d} \sigma .
\end{aligned}
$$

Since we have $\mu>1$ and on the boundary $\Sigma S_{N}^{i}=S_{N}^{e}$ and $\phi^{i}=\phi^{e}$, it follows that:

$$
\begin{aligned}
&\left|\phi-S_{N}^{e}\right|_{1, C \Omega}^{2} \leq\left|\phi-S_{N}^{e}\right|_{1, C \Omega}^{2}+\mu\left|\phi-S_{N}^{i}\right|_{1, \Omega}^{2} \\
&=\mu \int_{\Sigma}\left(\phi^{e}-S_{N}^{e}\right)\left\{\frac{\partial}{\partial n}\left(\phi^{i}-S_{N}^{i}\right)-\frac{1}{\mu} \frac{\partial}{\partial n}\left(\phi^{e}-S_{N}^{e}\right)\right\} \mathrm{d} \sigma .
\end{aligned}
$$

The term between the brackets reads:

$$
\begin{aligned}
\frac{\partial}{\partial n}\left(\phi^{i}-S_{N}^{i}\right)-\frac{1}{\mu} \frac{\partial}{\partial n}\left(\phi^{e}-S_{N}^{e}\right) & =\frac{\partial}{\partial n}\left(\phi^{i}-\frac{1}{\mu} \phi^{e}\right)-\frac{\partial}{\partial n}\left(S_{N}^{i}-\frac{1}{\mu} S_{N}^{e}\right) \\
& =-\left(\frac{1}{\mu}-1\right) g+\sum_{k=0}^{N}\left(\frac{1}{\mu^{k+1}} \frac{\partial \phi_{k}^{e}}{\partial n}-\frac{1}{\mu^{k}} \frac{\partial \phi_{k}^{i}}{\partial n}\right) \\
& =\sum_{k=2}^{N} \frac{1}{\mu^{k}}\left(\frac{\partial \phi_{k-1}^{e}}{\partial n}-\frac{\partial \phi_{k}^{i}}{\partial n}\right)+\frac{1}{\mu^{N+1}} \frac{\partial \phi_{N}^{e}}{\partial n} \\
& =\frac{1}{\mu^{N+1}} \frac{\partial \phi_{N}^{e}}{\partial n} .
\end{aligned}
$$

Now (23) together with (24) give

$$
\begin{aligned}
\left|\phi-S_{N}^{e}\right|_{1, \complement \Omega}^{2} & \leq \frac{1}{\mu^{N}} \int_{\Sigma}\left(\phi^{e}-S_{N}^{e}\right) \frac{\partial \phi_{N}^{e}}{\partial n} \mathrm{~d} \sigma \leq \frac{1}{\mu^{N}}\left\|\frac{\partial \phi_{N}^{e}}{\partial n}\right\|_{-\frac{1}{2}, \Sigma}\left\|\phi-S_{N}^{e}\right\|_{\frac{1}{2}, \Sigma} \\
& \leq \frac{C}{\mu^{N}}\|g\|_{-\frac{1}{2}, \Sigma}\left\|\phi-S_{N}^{e}\right\|_{\frac{1}{2}, \Sigma},
\end{aligned}
$$

where $C$ is a positive constant independent of $\mu$ and $\|\cdot\|_{\frac{1}{2}, \Sigma}$ denotes the norm in the Sobolev space $\mathbb{H}^{1 / 2}(\Sigma)$. We then deduce the estimate

$$
\left|\phi-S_{N}^{e}\right|_{1, C \Omega} \leq \frac{C_{e}}{\mu^{N}}\|g\|_{-\frac{1}{2}, \Sigma}
$$

where $C_{e}$ is a positive constant independent of $\mu$. In a similar way, we obtain

$$
\left|\phi-S_{N}^{i}\right|_{1, \Omega}^{2} \leq \frac{1}{\mu^{N+1}} \int_{\Sigma}\left(\phi^{i}-S_{N}^{i}\right) \frac{\partial \phi_{N}^{i}}{\partial n} \mathrm{~d} \sigma \leq \frac{C}{\mu^{N+1}}\|g\|_{-\frac{1}{2}, \Sigma}\left\|\phi-S_{N}^{i}\right\|_{\frac{1}{2}, \Sigma}
$$

and

$$
\left\|\phi-S_{N}^{i}\right\|_{1, \Omega} \leq \frac{C_{i}}{\mu^{N+1}}\|g\|_{-\frac{1}{2}, \Sigma}
$$

where $C_{i}$ is a positive constant independent of $\mu$.

\section{COMPUTATION OF THE MAGNETIC FIELD FROM THE RSP}

\subsection{Why adding the source field to the reaction field leads to inaccurate results?}

With the expansions (18) we can explain the reason of the cancellation and propose a way to circumvent it. Writing formula (15) for $\mathbf{H}_{\mathbf{m}}$ with the expression (18) for $\phi$, we get for 
$y \in \Omega$

$$
\begin{aligned}
\mathbf{H}_{\mathbf{m}}(y) & =-\frac{\mu-1}{\mu} \int_{\Sigma}\left(g(x) \nabla_{y} G(x, y)-\phi_{0}^{i}(x) \nabla_{y} G_{n}(x, y)\right) \mathrm{d} \sigma_{x} \\
+ & \frac{\mu-1}{\mu} \int_{\Sigma}\left(\sum_{k=1}^{+\infty} \frac{1}{\mu^{k}} \phi_{k}^{i}(x)\right) \nabla_{y} G_{n}(x, y) \mathrm{d} \sigma_{x} .
\end{aligned}
$$

Now, since $\phi_{0}^{i}$ is the solution of problem $\left(\mathcal{P}_{0}^{i}\right)$ we have for $y \in \Omega$

$$
\int_{\Sigma}\left(\frac{\partial \phi_{0}^{i}}{\partial n}(x) G(x, y)-\phi_{0}^{i}(x) G_{n}(x, y)\right) \mathrm{d} \sigma_{x}=\phi_{0}^{i}(y)
$$

and by differentiation with respect to $y$,

$$
\int_{\Sigma}\left(g(x) \nabla_{y} G(x, y)-\phi_{0}^{i}(x) \nabla_{y} G_{n}(x, y)\right) \mathrm{d} \sigma_{x}=\nabla_{y} \phi_{0}^{i}(y) .
$$

Thus (28) reads for $y \in \Omega$

$$
\mathbf{H}_{\mathbf{m}}(y)=-\frac{\mu-1}{\mu} \nabla \phi_{0}^{i}(y)+\frac{\mu-1}{\mu} \int_{\Sigma}\left(\sum_{k=1}^{+\infty} \frac{1}{\mu^{k}} \phi_{k}^{i}(x)\right) \nabla_{y} G_{n}(x, y) \mathrm{d} \sigma_{x} .
$$

On the other hand, the source field $\mathbf{H}_{\mathbf{s}}$ satisfies in the permeable region $\Omega$, see (2), the following equations:

$$
\left\{\begin{aligned}
\operatorname{div} \mathbf{H}_{\mathbf{s}} & =0 & & \text { in } \Omega \\
\operatorname{rot} \mathbf{H}_{\mathbf{s}} & =\mathbf{0} & & \text { in } \Omega \\
\left.\mathbf{H}_{\mathbf{s}}\right|_{\Omega} \cdot \mathbf{n} & =\left.\mathbf{H}_{\mathbf{s}}\right|_{C \Omega} \cdot \mathbf{n} & & \text { on } \Sigma
\end{aligned}\right.
$$

As $\mathbf{H}_{\mathbf{s}}$ is curl free in $\complement \Omega_{s}$, there exists a scalar function $\phi_{s}$ such that $\mathbf{H}_{\mathbf{s}}=\nabla \phi_{s}$ in $\complement \Omega_{s}$. From (32), we deduce that the function $\phi_{s}$ satisfies in $\Omega: \phi_{s} \in \mathbb{H}^{1}(\Omega)$ and

$$
\left\{\begin{array}{l}
\Delta \phi_{s}=0 \quad \text { in } \Omega, \\
\frac{\partial \phi_{s}}{\partial n}=\left.\mathbf{H}_{\mathbf{s}}\right|_{C \Omega} \cdot \mathbf{n} \quad \text { on } \Sigma .
\end{array}\right.
$$

It means that $\phi_{s}$ is a solution of problem $\left(\mathcal{P}_{0}^{i}\right)$ and therefore in $\bar{\Omega}$ we have $\phi_{s}=\phi_{0}^{i}+C$ where $C \in \mathbb{R}$ is a constant. Thus:

$$
\mathbf{H}_{\mathbf{s}}(y)=\nabla \phi_{0}^{i}(y) \quad \forall y \in \Omega .
$$

For $y \in \Omega$ we can then express $\mathbf{H}_{\mathbf{m}}$ as given by (31) as

$$
\mathbf{H}_{\mathbf{m}}(y)=-\mathbf{H}_{\mathbf{s}}(y)+\frac{1}{\mu} \mathbf{H}_{\mathbf{s}}(y)+\frac{\mu-1}{\mu} \int_{\Sigma}\left(\sum_{k=1}^{+\infty} \frac{1}{\mu^{k}} \phi_{k}^{i}(x)\right) \nabla_{y} G_{n}(x, y) \mathrm{d} \sigma_{x} .
$$

Finally the magnetic field $\mathbf{H}$ is given for $y \in \Omega$ by:

$$
\begin{aligned}
\mathbf{H}(y)= & \mathbf{H}_{\mathbf{m}}(y)+\mathbf{H}_{\mathbf{s}}(y) \\
= & \frac{1}{\mu} \mathbf{H}_{\mathbf{s}}(y)+\frac{\mu-1}{\mu} \int_{\Sigma}\left(\sum_{k=1}^{+\infty} \frac{1}{\mu^{k}} \phi_{k}^{i}(x)\right) \nabla_{y} G_{n}(x, y) \mathrm{d} \sigma_{x} \\
= & \frac{1}{\mu} \mathbf{H}_{\mathbf{s}}(y)+\frac{1}{\mu} \int_{\Sigma} \phi_{1}^{i}(x) \nabla_{y} G_{n}(x, y) \mathrm{d} \sigma_{x} \\
& +\sum_{k=1}^{+\infty}\left(\frac{1}{\mu^{k+1}} \int_{\Sigma}\left(\phi_{k+1}^{i}(x)-\phi_{k}^{i}(x)\right) \nabla_{y} G_{n}(x, y) \mathrm{d} \sigma_{x}\right) .
\end{aligned}
$$


We are now in position to understand why adding the source field $\mathbf{H}_{\mathbf{s}}$ to the field $\mathbf{H}_{\mathbf{m}}=$ $-\nabla \phi$ leads to inaccurate results inside $\Omega$. In (35) we have the asymptotic development of $\mathbf{H}_{\mathbf{m}}$ in power of $1 / \mu$. For large values of $\mu$, the leading term is $-\mathbf{H}_{\mathbf{s}}$ while the other terms are below the accuracy level of the computation. However, from (36) we understand that these other terms are the ones that give $\mathbf{H}$. So when we compute $\mathbf{H}$ by adding $\mathbf{H}_{\mathbf{s}}$ to $\mathbf{H}_{\mathbf{m}}$, we cancel the leading term in $\mathbf{H}_{\mathbf{m}}$ and loose the relevant numerical values.

\subsection{Comparison with the two potential method}

Let us now investigate the link between the classical two potential method and the above asymptotic expansion. For the two potential method we consider the case where the total magnetic potential $\psi$ is used in $\Omega$ and the RSP $\phi$ elsewhere. The magnetostatic problem is formulated in term of these two scalar potentials as follows:

$$
\begin{cases}\Delta \psi=0 & \text { in } \Omega, \\ \Delta \phi=0 & \text { in } C \Omega, \\ \frac{\partial \phi}{\partial n}-\mu \frac{\partial \psi}{\partial n}=g & \text { on } \Sigma,\end{cases}
$$

with a jump between $\psi$ and $\phi$ on $\Gamma$ given by, see relation (9),

$$
\phi(x)-\psi(x)=\int_{\gamma} \mathbf{H}_{\mathbf{s}} \cdot \mathbf{d t}
$$

where $\gamma$ denotes a path on $\Sigma$ from a given fixed point $x_{0}$ on $\Sigma$ where $\phi\left(x_{0}\right)=\psi\left(x_{0}\right)$ to any general point $x$ on $\Sigma$. As $\mathbf{H}_{\mathbf{s}}$ is curl free in $C \Omega_{s}$, there exists a scalar function $\phi_{s}$ such that $\mathbf{H}_{\mathbf{s}}=\nabla \phi_{s}$ in $\complement \Omega_{s}$, see (33). This implies that

$$
\phi(x)-\psi(x)=\phi_{s}(x)-\phi_{s}\left(x_{0}\right)
$$

and $\phi_{s}$ can be chosen so that $\phi_{s}\left(x_{0}\right)=0$.

The two potential method can therefore be formulated in the following way: find $\chi$ such that

$$
\begin{cases}\Delta \chi=0 & \text { in } \Omega \text { and } C \Omega \\ \left.\frac{\partial \chi}{\partial n}\right|_{C \Omega}-\left.\mu \frac{\partial \chi}{\partial n}\right|_{\Omega}=g & \text { on } \Sigma \\ \left.\chi\right|_{C \Omega}-\left.\chi\right|_{\Omega}=\phi_{s} & \text { on } \Sigma\end{cases}
$$

where $\left.\chi\right|_{\Omega}$ represents the total magnetic potential in $\Omega,\left.\chi\right|_{\mathrm{C} \Omega}$ the $\mathrm{RSP}, \phi_{s} \in \mathbb{H}^{1 / 2}(\Sigma)$ is a scalar potential of the source field $\mathbf{H}_{\mathbf{S}}$ and $g=\mathbf{H}_{\mathbf{S}} \cdot \mathbf{n} \in \mathbb{H}^{-1 / 2}(\Sigma)$.

We get the weak form of problem (40) using a procedure analogous to the one presented in section 2.1. It reads: find $\chi \in \mathbb{L}^{2}\left(\mathbb{R}^{3}\right)$ with $\left.\chi\right|_{\Omega} \in \mathbb{H}^{1}(\Omega)$ and $\left.\chi\right|_{\mathrm{C} \Omega} \in \mathbb{W}^{1}(\complement \Omega)$ such that $\left.\chi\right|_{\mathrm{C} \Omega}-\left.\chi\right|_{\Omega}=\phi_{s}$ on $\Sigma$ and

$$
\mu \int_{\Omega} \nabla \chi \cdot \nabla \varphi \mathrm{d} x+\int_{\complement \Omega} \nabla \chi \cdot \nabla \varphi \mathrm{d} x=-\int_{\Sigma} g \varphi \mathrm{d} \sigma \quad \forall \varphi \in \mathbb{W}^{1}\left(\mathbb{R}^{3}\right) .
$$

In (41) the solution $\chi$ and the test functions $\varphi$ belong to different spaces. To overcome this limitation, let us consider the substitution $\chi=\xi+\chi_{s}$ where $\chi_{s} \in \mathbb{L}^{2}\left(\mathbb{R}^{3}\right)$ with $\left.\chi_{s}\right|_{\Omega} \in \mathbb{H}^{1}(\Omega)$ and $\left.\chi_{s}\right|_{\mathcal{C} \Omega} \in \mathbb{W}^{1}(\complement \Omega)$ satisfies the condition $\left.\chi_{s}\right|_{\mathrm{C} \Omega}-\left.\chi_{s}\right|_{\Omega}=\phi_{s}$ on $\Sigma$. We get 
the following equivalent variational formulation for problem (40): find $\xi \in \mathbb{W}^{1}\left(\mathbb{R}^{3}\right)$ such that $\forall \varphi \in \mathbb{W}^{1}\left(\mathbb{R}^{3}\right)$

$$
\mu \int_{\Omega} \nabla \xi \cdot \nabla \varphi \mathrm{d} x+\int_{\complement \Omega} \nabla \xi \cdot \nabla \varphi \mathrm{d} x=-\int_{\Sigma} g \varphi \mathrm{d} \sigma-\mu \int_{\Omega} \nabla \chi_{s} \cdot \nabla \varphi \mathrm{d} x-\int_{C \Omega} \nabla \chi_{s} \cdot \nabla \varphi \mathrm{d} x .
$$

From the Lax-Milgram theorem one can prove that problem (42) has a unique solution in the Sobolev space $\mathbb{W}^{1}\left(\mathbb{R}^{3}\right)$, see $[9]$.

Let us now consider the functions $\chi^{i} \in \mathbb{H}^{1}(\Omega)$ and $\chi^{e} \in \mathbb{W}^{1}(C \Omega)$ defined by

$$
\chi^{i}=-\phi_{0}^{i}\left(x_{0}\right)+\sum_{k=1}^{+\infty} \frac{1}{\mu^{k}} \phi_{k}^{i} \quad \text { and } \quad \chi^{e}=\sum_{k=0}^{+\infty} \frac{1}{\mu^{k}} \phi_{k}^{e}
$$

where the functions $\phi_{k}^{i} \in \mathbb{H}^{1}(\Omega)$ and $\phi_{k}^{e} \in \mathbb{W}^{1}(\complement \Omega)$ are solutions of the coupled problems $\left(\mathcal{P}_{k}^{i}\right)$ and $\left(\mathcal{P}_{k}^{e}\right)$. We have $\Delta \chi^{i}=0$ in $\Omega, \Delta \chi^{e}=0$ in $\complement \Omega$ and $\frac{\partial \chi^{e}}{\partial n}-\mu \frac{\partial \chi^{i}}{\partial n}=g$ on $\Sigma$. Moreover $\chi^{e}-\chi^{i}=-\phi_{0}^{i}\left(x_{0}\right)+\phi_{0}^{e}=-\phi_{0}^{i}\left(x_{0}\right)+\phi_{0}^{i}$ on $\Sigma$ and from (33) $\phi_{s}=\phi_{0}^{i}+C$ where $C$ is a constant given by $C=-\phi_{0}^{i}\left(x_{0}\right)$ since $\phi_{s}\left(x_{0}\right)=0$. It follows that $\chi^{e}-\chi^{i}=\phi_{s}$ and since problem (40) has a unique solution we can conclude that relations (43) give the RSP and the total scalar potential in the two potential method in terms of the potentials involved in our asymptotic method.

\subsection{Computation of the magnetic field using the asymptotic method}

Details on the finite element implementation of the two potential method can be found for instance in [1], [10], [11], [3] or [12]. A drawback of the two potential method is that the unknown $\chi$ is a discontinuous function across the interface $\Sigma$ and the method require the computation of the line integral (38). To avoid these deficiencies a formulation involving two scalar potentials which must be evaluated sequentially has been propound in [13]. The first potential is calculated under the assumption that the magnetic permeability of the ferromagnetic domain $\Omega$ is infinite and a fictitious magnetic field is obtained. Then an unknown field which is defined as the difference between the real magnetic field and the fictitious magnetic field is considered and the total scalar potential associated with the difference field is introduced. This potential is continuous in the whole space and enables the computation of the real magnetic field within the ferromagnetic domain without any cancellation error.

As a by-product of the study presented in section 2 we give another way to compute the magnetic field $\mathbf{H}$ within a region with high magnetic permeability without introducing the total magnetic potential. For $\mu$ in the range $\left[10^{2}, 10^{4}\right]$, relation (36) shows that $\mathbf{H}$ can be approximated by

$$
\begin{aligned}
\mathbf{H}(y) \approx & \frac{1}{\mu} \mathbf{H}_{\mathbf{s}}(y)+\frac{1}{\mu} \int_{\Sigma} \phi_{1}^{i}(x) \nabla_{y} G_{n}(x, y) \mathrm{d} \sigma_{x} \\
& +\frac{1}{\mu^{2}} \int_{\Sigma}\left(\phi_{2}^{i}(x)-\phi_{1}^{i}(x)\right) \nabla_{y} G_{n}(x, y) \mathrm{d} \sigma_{x} .
\end{aligned}
$$

Relation (44) gives a way to compute $\mathbf{H}$ once $\phi_{1}^{i}$ and $\phi_{2}^{i}$ are known. To compute $\phi_{1}^{i}$, and $\phi_{2}^{i}$ we can follow the steps presented in the proof of proposition 1 . We have to solve the three Neumann problems $\left(\mathcal{P}_{0}^{i}\right),\left(\mathcal{P}_{1}^{i}\right)$, and $\left(\mathcal{P}_{2}^{i}\right)$ in the bounded domain $\Omega$ and the two Dirichlet problems $\left(\mathcal{P}_{0}^{e}\right)$ and $\left(\mathcal{P}_{1}^{e}\right)$ in the exterior domain $\complement \Omega$.

Problem $\left(\mathcal{P}_{0}^{i}\right)$ can be solved using the finite element method on the bounded domain $\Omega$. Its solution is unique up to a constant $C_{0}$ and the numerically computed solution is an 
approximation of $\widetilde{\phi_{0}^{i}}$ where $\widetilde{\phi_{0}^{i}}=\phi_{0}^{i}+C_{0}$. As shown in the proof of proposition 1 , we have

$$
C_{0}=-\left(\int_{\Sigma} \frac{\partial \widetilde{\phi}_{0}^{e}}{\partial n} \mathrm{~d} \sigma\right) \times\left(\int_{\Sigma} \frac{\partial v}{\partial n} \mathrm{~d} \sigma\right)^{-1}
$$

where $v \in \mathbb{W}^{1}(\complement \Omega)$ is given by

$$
\left(\mathcal{P}_{v}\right) \quad \begin{cases}\Delta v=0 & \text { in } \quad \complement \Omega, \\ v=1 & \text { on } \Sigma,\end{cases}
$$

and $\widetilde{\phi}_{0}^{e}$ is given by

$$
\left(\widetilde{\mathcal{P}}_{0}^{e}\right) \quad\left\{\begin{array}{lll}
\Delta \widetilde{\phi}_{0}^{e}=0 & \text { in } \quad C \Omega, \\
\widetilde{\phi}_{0}^{e}=\widetilde{\phi}_{0}^{i} & \text { on } \quad \Sigma .
\end{array}\right.
$$

Problems $\left(\mathcal{P}_{v}\right)$ and $\left(\widetilde{\mathcal{P}}_{0}^{e}\right)$ involve Laplace equation with Dirichlet boundary conditions on the exterior domain $C \Omega$. We refer to [14] for a review of numerical methods for solving such problems on unbounded domains using a finite element approach. Typically one truncates the domain and sets artificial boundary conditions on the external boundary of the bounded computational domain. The solution to $\left(\mathcal{P}_{0}^{e}\right)$ is then given by $\phi_{0}^{e}=\widetilde{\phi}_{0}^{e}+C_{0} v$. As shown before, the compatibility condition for problem $\left(\mathcal{P}_{1}^{i}\right)$ is automatically satisfied. Computation of $\phi_{1}^{i}$ and $\phi_{1}^{e}$ and then computations of $\phi_{2}^{i}$ are achieved in the same way. The approach described here is efficient since the whole computation reduces to solve sequentially two Laplace equations, one in $\Omega$ and one in $\complement \Omega$ with several right-hand sides.

The numerical results presented in section 4 were obtained following this computation procedure. Problems $\left(\mathcal{P}_{k}^{i}\right)$ in the ferromagnetic core can be solved using the finite element method. The matrix of the discretized problem is the same for all the $\left(\mathcal{P}_{k}^{i}\right)$ and therefore time is saved in the matrix assembling process. Furthermore if a direct method is used to solve the linear system then only one matrix factorization is required. If an iterative method is used, depending on the preconditioning technique used this advantage can be lost. Problems $\left(\mathcal{P}_{k}^{e}\right)$ are set in an unbounded domain. The method we used to compute $\phi_{k}^{e}$ is discussed in [15]. An artificial boundary is introduced at a close distance from the ferromagnetic core and the behavior of $\phi_{k}^{e}$ at infinity is handle through an exact boundary condition which is set on this artificial boundary. It has the advantage of greatly reducing the size of the domain to be mesh and therefore the size of the linear system. The boundary can be set very closely to $\Omega$ since the only relevant information for the following computations are the values of $\phi_{k}^{e}$ on the boundary $\Sigma$. One advantage in using the method presented in [15] is that it enables the computation of the magnetic field $\mathbf{H}$ in any point of the space through relation (44) without numerical differentiation.

So far we have assumed for simplicity that the metallic core $\Omega$ has a constant relative magnetic permeability $\mu$. This assumption is not a limitation of the study and the general case of a domain $\Omega$ with piecewise constant permeabilities could be studied in a similar way but involves cumbersome notations.

\section{NUMERICAL ILLUSTRATIONS}

\subsection{A test case}

In order to illustrate our discussion we consider the case where the domain $\Omega$ is a ball of radius $R=1 \mathrm{~cm}$ and of relative magnetic permeability $\mu$. The source field $\mathbf{H}_{\mathbf{s}}$ is 


\begin{tabular}{|c|c|c|c|c|c|}
\hline$\mu$ & $\|\mathbf{H}\|_{2}$ exact & $\|\mathbf{H}\|_{2}$ using 1 term & error (\%) & $\|\mathbf{H}\|_{2}$ using 2 terms & error (\%) \\
\hline 10 & $0.183510^{6}$ & $0.215710^{6}$ & 16.2 & $0.210710^{6}$ & 13.8 \\
$10^{2}$ & $0.231610^{5}$ & $0.234910^{5}$ & 1.4 & $0.234310^{5}$ & 1.2 \\
$10^{3}$ & $0.237810^{4}$ & $0.237010^{4}$ & 0.32 & $0.237010^{4}$ & 0.32 \\
$10^{4}$ & $0.238410^{3}$ & $0.237210^{3}$ & 0.50 & $0.237210^{3}$ & 0.50 \\
\hline
\end{tabular}

Table 2: Comparison of the magnitude of $\mathbf{H}$ in $\Omega$ computed analytically and using (46), (47).

assumed to be constant in intensity and direction $\left(H_{s}=0.79510^{6} \mathrm{H} / \mathrm{m}\right)$ so that the exact expression for the magnetic field $\mathbf{H}$ is known. In the domain $\Omega$ the magnetic field $\mathbf{H}$ is constant and given by, see [16],

$$
\mathbf{H}=\frac{3}{\mu+2} \mathbf{H}_{\mathbf{s}} .
$$

The values for $\mathbf{H}$ computed over $\Omega$ by adding the two vector fields $\mathbf{H}_{\mathbf{s}}$ and $\mathbf{H}_{\mathbf{m}}$ are shown in table 1. Namely we give the magnitude of $\mathbf{H}$ computed by adding the two fields $\mathbf{H}_{\mathbf{s}}$ and $\mathbf{H}_{\mathbf{m}}$ and computed analytically for different values of the magnetic permeability $\mu$ for an arbitrary point chosen in $\Omega$. The field $\mathbf{H}_{\mathbf{m}}$ is computed from the RSP by relation (15) with a quadratic error smaller than $1 \%$. The numerical implementation is achieved using the program MÉLina [17] developed at the Institut de Recherche Mathématique de Rennes, University of Rennes 1. As mentioned before, the accuracy in the values of the magnetic field $\mathbf{H}$ decreases when the magnetic permeability $\mu$ increases due to the cancellation phenomenon. The components of the two quantities $\mathbf{H}_{\mathbf{s}}$ and $\mathbf{H}_{\mathbf{m}}$ have nearly the same magnitude with opposite signs and thus computation errors dominate when the two fields are added.

In table 2 we show the values of $\mathbf{H}$ computed using relation (36). Namely we give for several values of $\mu$ the magnitude of $\mathbf{H}$ in $\Omega$ computed using one term in the expansion (36),

$$
\mathbf{H}(y) \approx \frac{1}{\mu} \mathbf{H}_{\mathbf{s}}(y)+\frac{1}{\mu} \int_{\Sigma} \phi_{1}^{i}(x) \nabla_{y} G_{n}(x, y) \mathrm{d} \sigma_{x}
$$

and two terms,

$$
\begin{aligned}
\mathbf{H}(y) \approx & \frac{1}{\mu} \mathbf{H}_{\mathbf{s}}(y)+\frac{1}{\mu} \int_{\Sigma} \phi_{1}^{i}(x) \nabla_{y} G_{n}(x, y) \mathrm{d} \sigma_{x} \\
& +\frac{1}{\mu^{2}} \int_{\Sigma}\left(\phi_{2}^{i}(x)-\phi_{1}^{i}(x)\right) \nabla_{y} G_{n}(x, y) \mathrm{d} \sigma_{x} .
\end{aligned}
$$

The three Neumann problems $\left(\mathcal{P}_{0}^{i}\right),\left(\mathcal{P}_{1}^{i}\right)$, and $\left(\mathcal{P}_{0}^{i}\right)$ in the bounded domain $\Omega$ are solved using the $\mathcal{P}_{2}$ Lagrange finite element method. To solve the two Dirichlet problems $\left(\mathcal{P}_{0}^{e}\right)$ and $\left(\mathcal{P}_{1}^{e}\right)$ in the exterior domain $C \Omega$ we couple the finite element method with a boundary integral method as described in [15].

It can be seen that for $\mu$ in the range $\left[10^{2}, 10^{4}\right]$ formula (46) gives accurate results for the magnetic field $\mathbf{H}$. Using two terms in the asymptotic expansion does not improve significantly the accuracy: the term in $1 / \mu^{2}$ falls under the accuracy of the numerical method used to compute $\mathbf{H}_{\mathbf{m}}$ from the RSP. 


\subsection{Example of an electromagnet}

We consider an electromagnet which consists of a cylindrical core situated inside a pair of coils, see figure 1. The currents in the two coils are imposed in same direction and of constant density $\left(1 \mathrm{~A} / \mathrm{mm}^{2}\right)$. The electromagnet core has the following physical and geometric features: diameter: $1.5 \mathrm{~cm}$, length: $4 \mathrm{~cm}$, magnetic permeability $\mu=10^{3}$. We have bounded the domain with a sphere of radius $3 \mathrm{~cm}$ and have meshed the bounded domain $\Omega_{\Gamma}$ with 56160 tetrahedral elements, see figure 2 . We couple the finite element method with a boundary integral method as described in [15] to compute the first three terms $\phi_{0}^{i}, \phi_{1}^{i}$ and $\phi_{2}^{i}$ in the asymptotic expansion of the RSP. The isolines for the RSP inside the core are depicted in figure 2. The magnetic field in the core is then computed using relation (47). The whole computation, done on a desktop computer (Pentium IV $3 \mathrm{Ghz}$ ), required $166 \mathrm{~s}$. Figure 3 shows the total magnetic field $\mathbf{H}$ inside the core.

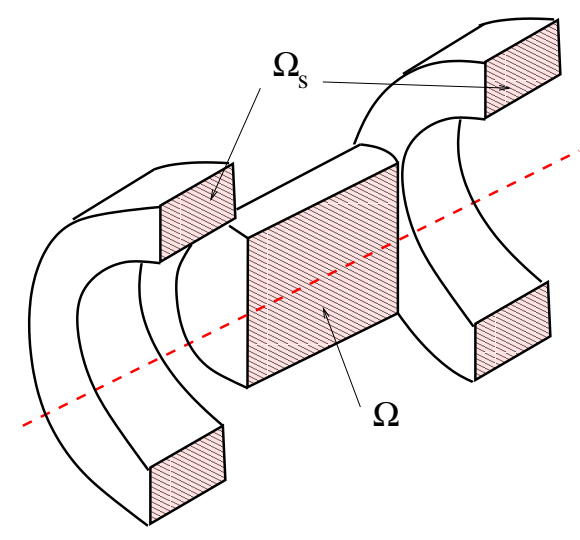

Figure 1: Example of the electromagnet under consideration.
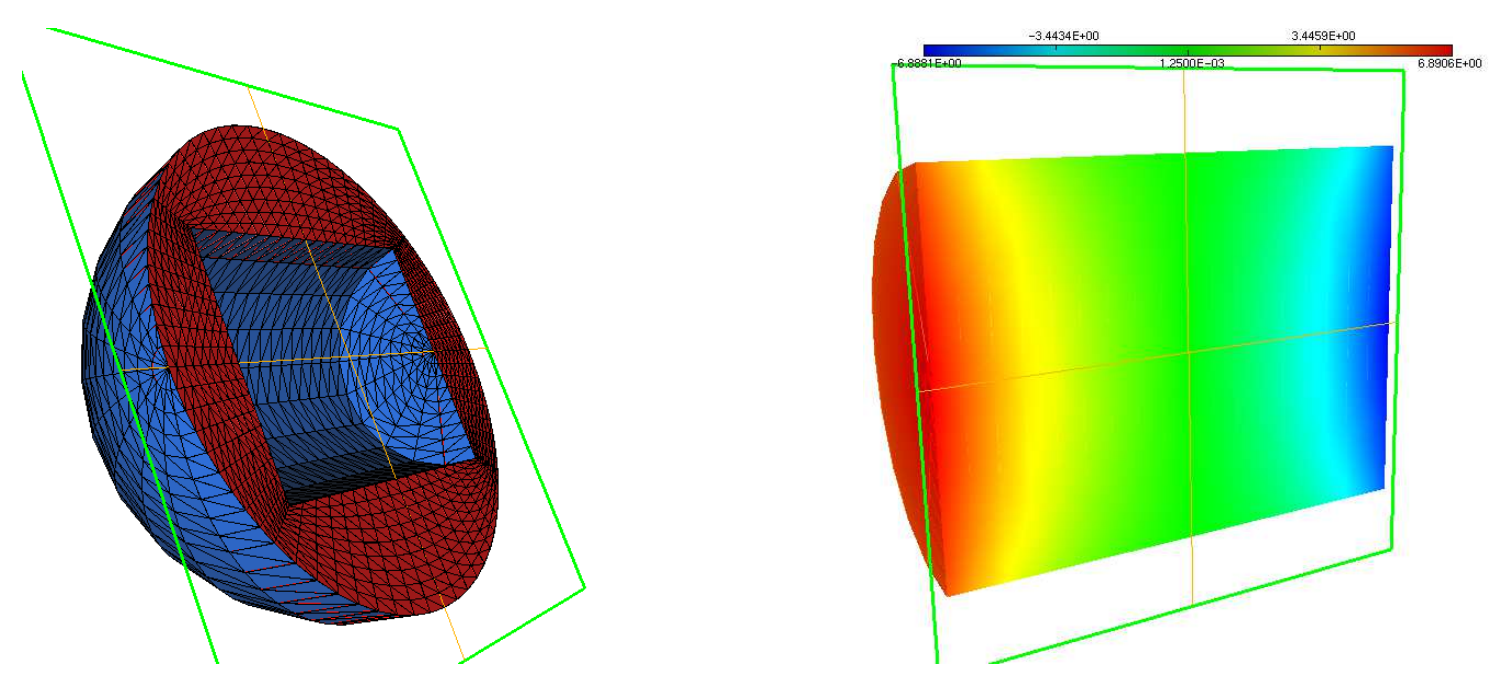

Figure 2: Visualisation of the mesh of the computational domain $\Omega_{\Gamma}$ (left) and isovalues for the RSP inside the core (right) on the plane depicted in fig. 1. 


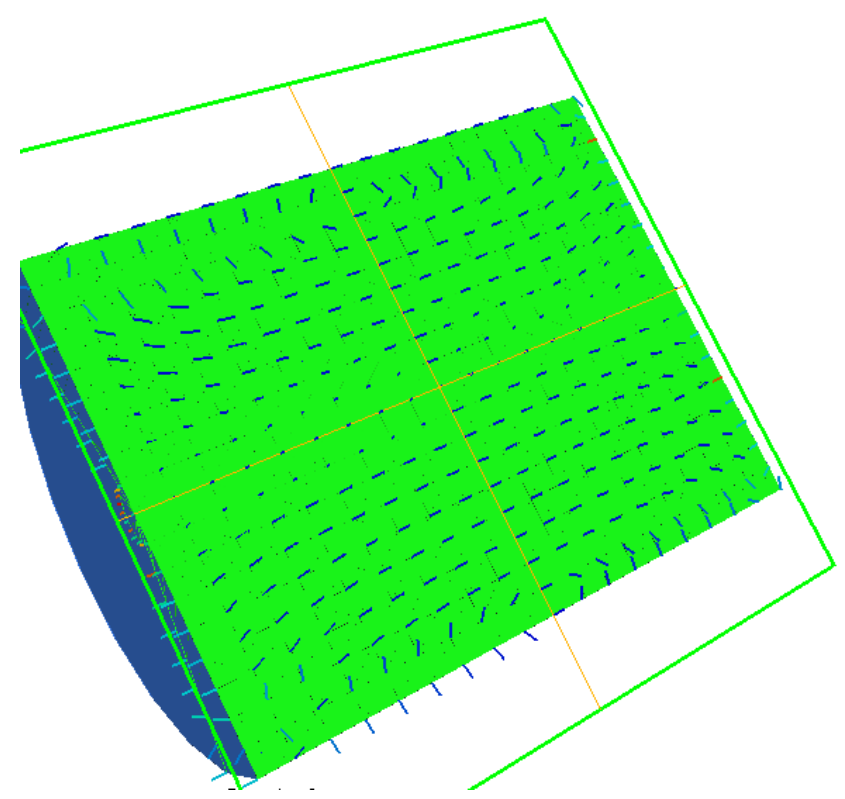

Figure 3: Total magnetic field $\mathbf{H}$ (right) inside the core on the plane depicted in fig. 1.

\section{CONCLUSION}

Usually to solve three-dimensional magnetic field problems, the use of both the reduced scalar potential and the total scalar potential is recommended since the use of the reduced scalar potential $\phi$ alone gives severe loss of accuracy in the regions with high magnetic permeability. Firstly we explained the reasons for this lack of accuracy. For large $\mu$ the numerical values of the field $\mathbf{H}_{\mathbf{m}}=-\nabla \phi$ are not precise enough to get the total magnetic field $\mathbf{H}$ by adding $\mathbf{H}_{\mathbf{m}}$ to the source field $\mathbf{H}_{\mathbf{s}}$ since $\mathbf{H}_{\mathbf{m}}$ and $\mathbf{H}_{\mathbf{s}}$ cancel each other out. We were able to analyze the cancellation in the asymptotic expansions of $\mathbf{H}_{\mathbf{m}}$ in power of $1 / \mu$. Moreover we have shown that the use of the two potentials is not necessarily required and we have presented a method to accurately compute the magnetic field $\mathbf{H}$ in the permeable region through an asymptotic expansion of $\mathbf{H}$ in power of $1 / \mu$.

\section{References}

[1] Simkin J, Trowbridge CW. On the use of the total scalar potential in the numerical solution of field problems in electromagnetics. Int. J. Numer. Methods Engin. 1979, 14:423-440.

[2] A. Bossavit, C. Emson, and I.D. Mayergoyz. Méthodes numériques en électromagnétisme. Eyrolles, 1991.

[3] Sussman M. Remarks on computational magnetostatics. Int. J. Numer. Methods Engin. 1988, 26:987-1000.

[4] Ciric IR. Formulas for the magnetic field of polygonal cross section current coils. IEEE Transactions on Magnetics 1992, 28:1064-1067.

[5] Fontana M. Integration methods for the calculation of the magnetostatic field due to coils. Technical Report 2001-07, Chalmers Finite Element Center, 2001. 
[6] Jin JM. The finite element method in electromagnetics. Wiley:New York, 1993.

[7] Chen G, Zhou J. Boundary element methods. Academic Press, 1992.

[8] Omeragic D, Silvester PP. Numerical differentiation in magnetic field postprocessing. International Journal of Numerical Modelling 1996, 9:99-113.

[9] Dautray R, Lions JL. Mathematical Analysis and Numerical Methods for Science and Technology. Spinger Verlag, 1990.

[10] Simkin J Trowbridge CW. Three-dimensional nonlinear electromagnetic field computations, using scalar potentials. IEE PROC 1980, 127(6):368-374.

[11] Pissanetzky S. Solution of three-dimensional, anisotropic, nonlinear problems of magnetostatics using two scalar potentials, finite and infinite multipolar elements and automatic mesh generation. IEEE Transactions on Magnetics 1982, 18(2):346- 350.

[12] Rodger D. Implementation of the total and reduced magnetic scalar potential technique usinh hierachical finite elements. J. Appl. Phys. 1991, 69(8):5029-5031.

[13] Mayergoyz I, Chari M, D'Angelo J. A new scalar potential formulation for three-dimensional magnetostatic problems. IEEE Transactions on Magnetics 1987, 23(6):3889- 3894 .

[14] Tsynkov SV. Numerical solution of problems on unbounded domains. A review. Applied Numerical Mathematics 1998, 27(4):465-532.

[15] Balac S, Caloz G. Magnetostatic field computations based on the coupling of finite element and integral representation methods. IEEE Transactions on Magnetics 2002, 38:393-396.

[16] Jackson JD. Classical Electrodynamics. Wiley: New-York, 1998.

[17] Martin D. Mélina documentation, 2003. http://perso.univ-rennes1.fr/daniel. martin/melina [25 October 2006]

[18] Coco S, Ragusa C. Accurate computation of local and global electrostatic quantities from FE solutions. IEEE Transactions on Magnetics 2000, 36:732-735. 